\title{
Gaining the Upper Hand on Systemic Sclerosis Digital Ulcers
}

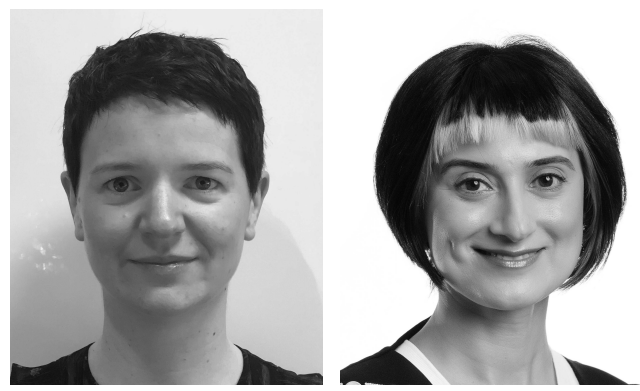

Vasculopathy of the small blood vessels is one of the cardinal features of systemic sclerosis (SSc). The anatomical alterations of the microcirculation and small blood vessels associated with Raynaud phenomenon, the most common vascular manifestation of $\mathrm{SSc}$, in combination with endothelial dysregulation and altered coagulation and fibrinolysis can lead to digital ulcers (DU) ${ }^{1,2}$. DU are a severe manifestation of SSc-associated vasculopathy, affecting up to half of patients with SSc, and are associated with a more fulminant SSc disease course ${ }^{1,3}$.

Current treatment options for DU remain inadequate, and DU continue to cause a large degree of pain and disability for patients with $\mathrm{SSc}^{1}$. Two randomized controlled trials (RCT) have supported the use of intravenous iloprost in the treatment of active $\mathrm{DU}^{4,5}$, and current treatment recommendations suggest that phosphodiesterase 5 inhibitors may be efficacious in the treatment of $\mathrm{DU}^{6}$.

The success of endothelin receptor antagonists (ERA) in the treatment of pulmonary arterial hypertension, another severe vasculopathic manifestation of SSc, triggered interest in their use for the treatment of DU. The RAPIDS-1 and RAPIDS-2 RCT evaluated the effect of bosentan on DU prevention and healing. In both studies, bosentan reduced the number of new DU, and the treatment effects appeared to be most pronounced in those patients with $>4 \mathrm{DU}$ at baseline $\mathrm{e}^{7,8}$. In these trials, the diagnosis of new DU and assessment of DU healing were determined by clinician assessment. Macitentan was later evaluated as a preventive therapy for DU in the DUAL-1 and -2 trials ${ }^{9}$. The DUAL-2 RCT was stopped early because of a lack of treatment efficacy, calling into question the efficacy of ERA in the treatment of DU. Similar to the RAPIDS RCT, DUAL- 1 and -2 used the physician-assessed number of new DU as the primary outcome. While the results of the DUAL trials may indicate a true lack of benefit of macitentan in the treatment of DU, the lack of treatment efficacy demonstrated may have resulted from the lack of a clear classification system of DU and high interobserver variability in the assessment of $\mathrm{DU}^{9}$. There remains inadequate consensus as to the very definition of a DU and the spectrum of finger lesions that should be considered DU in a clinical trial. The absence of a validated and reliable outcome measure for the assessment of DU continues to limit therapeutic trials of novel therapies.

To address this need, in this issue of The Journal, Bruni, et al present the Digital Ulcer Clinical Assessment Score (DUCAS) in systemic sclerosis ${ }^{10}$. This outcome measure, designed for use in therapeutic trials, is the first composite measure developed to clinically assess DU. Using consensus methodology, drawing upon the knowledge and experience of 8 experts in SSc-associated DU, the authors identified 7 domains important in the assessment of the burden of DU (Bruni, et al, Table 1$)^{10}$. The final score is weighted across all 7 domains, with the weighting of each item determined by expert consensus, to give a total possible score of 19.5 .

As a first step toward the validation of DUCAS according to the Outcome Measures in Rheumatology (OMERACT) Filter of truth, discrimination, and feasibility ${ }^{11}$, Bruni, et al have performed a small validation study of 44 patients from a single center, testing the content and construct validity of DUCAS ${ }^{10}$. The convergent and divergent correlation coefficients of DUCAS with other clinical outcome measures were evaluated, including DU-specific patient-reported outcomes (PRO). Content and construct validity were partially demonstrated in this study by showing that DUCAS had an appropriate correlation with physician-assessed global DU severity, PRO, as well as scores of hand disability and function. The DUCAS correlated most strongly with the physician global assessment of DU severity ( $\mathrm{r} 0.63, \mathrm{p}<0.001$ ). The authors also suggest that the DUCAS is a feasible measure of DU, because the assessment tool was successfully applied in this study during a single clinical visit.

A limitation of this initial validation study is the small number of patients enrolled. While the authors are to be congratulated on performing this initial testing of the performance of the DUCAS in a population of patients not dissimilar to larger international cohorts, it is not yet known whether these results are applicable to patients with SSc at large.

Any clinical assessment of DU is limited by the lack of an internationally accepted definition of DU and the poor interobserver variability of physician assessment of $\mathrm{DU}^{12,13}$.

See SSc ulcer outcome measure, page 603 
Assessment of both the presence and number of new DU combines to account for a maximum possible score of 4 out of a total 19.5 points of the DUCAS (Bruni, et al, Table 1$)^{10}$. All DUCAS in the study by Bruni, et al were calculated by a single physician at a single study visit. Moderate correlation of DUCAS with physician-assessed DU severity was demonstrated; however, the intra- and interobserver variability of DUCAS remain unknown. Careful assessment of these measures of agreement will be required in future studies.

One of the next key steps in the validation of DUCAS will be to test the score's responsiveness to change over time and its discriminatory capacity. There are a number of large, international SSc research collaborations including the European Scleroderma Trials and Research Group, and the Scleroderma Clinical Trials Consortium, which can be called upon to assist in the testing of DUCAS within longitudinal cohorts of patients. The use of DUCAS as an exploratory or secondary endpoint in clinical trials will serve to determine not only the discriminatory capacity of the score but also a minimum clinically important change in DUCAS, further validating it as an endpoint in future therapeutic trials.

It is likely that the performance of DUCAS would be improved with the adoption of a single consensus definition of DU and consistency regarding the hand lesions considered to be DU in clinical trials. Lesions such as skin fissures and digital pitting scars, and the classification of gangrene remain contentious issues ${ }^{12,13}$. Uniform classification criteria of DU are required to improve the quality of research in this important area. The consistency of the assessment of other manifestations of SSc in RCT, such as calculation of the modified Rodnan Skin Score, has been improved by providing clinicians with targeted training, thereby improving the quality of therapeutic trials ${ }^{14}$. In the future, it will be important to assess whether specific training in the application of tools such as DUCAS can improve the quality of the assessment of DU in RCT.

The DUCAS lays the foundation for a future of more nuanced assessment of DU in SSc. The limitations of a dichotomous "healed versus non-healed" paradigm for evaluating the efficacy of novel treatments have been demonstrated in many large studies. Given the morbidity and mortality associated with severe peripheral vascular disease in SSc, there is a pressing need to find effective therapies for patients. Effective future clinical trials will not be possible without the use of fully validated outcome measures.

LAURA ROSS ${ }^{\circledR}$, MBBS, FRACP,

MANDANA NIKPOUR, MBBS, FRACP, FRCPA, PhD,

Department of Rheumatology,

St Vincent's Hospital Melbourne,

and Department of Medicine,

The University of Melbourne,

Fitzroy, Australia.

Address correspondence to Assoc. Prof. M. Nikpour, Departments of

Rheumatology and Medicine, The University of Melbourne at St Vincent's
Hospital (Melbourne), 41 Victoria Parade, Fitzroy VIC, 3065, Australia E-mail:m.nikpour@unimelb.edu.au

LR is supported by a Musculoskeletal Australia PhD Scholarship and an Australian Government Research Training Program Scholarship. MN holds a National Health and Medical Research Council of Australia Fellowship (APP 1126370)

\section{REFERENCES}

1. Matucci-Cerinic M, Krieg T, Guillevin L, Schwierin B, Rosenberg D, Cornelisse P, et al. Elucidating the burden of recurrent and chronic digital ulcers in systemic sclerosis: long-term results from the DUO Registry. Ann Rheum Dis 2016;75:1770-6.

2. Allanore Y, Distler O, Matucci-Cerinic M, Denton CP. Review: defining a unified vascular phenotype in systemic sclerosis. Arthritis Rheum 2018;70:162-70.

3. Mihai C, Landewe R, van der Heijde D, Walker UA, Constantin PI, Gherghe AM, et al. Digital ulcers predict a worse disease course in patients with systemic sclerosis. Ann Rheum Dis 2016;75:681-6.

4. Wigley FM, Seibold JR, Wise RA, McCloskey DA, Dole WP. Intravenous iloprost treatment of Raynaud's phenomenon and ischemic ulcers secondary to systemic sclerosis. J Rheumatol 1992;19:1407-14.

5. Wigley FM, Wise RA, Seibold JR, McCloskey DA, Kujala G, Medsger TA Jr., et al. Intravenous iloprost infusion in patients with Raynaud's phenomenon secondary to systemic sclerosis. A multicenter, placebo-controlled, double-blind study. Ann Intern Med 1994;120:199-206.

6. Kowal-Bielecka O, Fransen J, Avouac J, Becker M, Kulak A, Allanore Y, et al. Update of EULAR recommendations for the treatment of systemic sclerosis. Ann Rheum Dis 2017;76:1327-39.

7. Korn JH, Mayes M, Matucci Cerinic M, Rainisio M, Pope J, Hachulla E, et al. Digital ulcers in systemic sclerosis: Prevention by treatment with bosentan, an oral endothelin receptor antagonist. Arthritis Rheum 2004;50:3985-93.

8. Matucci-Cerinic M, Denton CP, Furst DE, Mayes MD, Hsu VM, Carpentier P, et al. Bosentan treatment of digital ulcers related to systemic sclerosis: results from the RAPIDS-2 randomised, double-blind, placebo-controlled trial. Ann Rheum Dis 2010; 70:32-8.

9. Khanna D, Denton CP, Merkel PA, Krieg T, Le Brun FO, Marr A, et al. Effect of macitentan on the development of new ischemic digital ulcers in patients with systemic sclerosis: DUAL-1 and DUAL-2 randomized clinical trials. JAMA 2016;315:1975-88.

10. Bruni C, Ngcozana T, Braschi F, Pucci T, Piemonte G, Benelli L, et al. Preliminary validation of the digital ulcer clinical assessment score (DUCAS) in systemic sclerosis. J Rheumatol 2019;46:603-8.

11. Boers M, Brooks P, Strand CV, Tugwell P. The OMERACT Filter for outcome measures in rheumatology. J Rheumatol 1998; 25:198-9.

12. Hughes M, Tracey A, Bhushan M, Chakravarty K, Denton CP, Dubey S, et al. Reliability of digital ulcer definitions as proposed by the UK Scleroderma Study Group: A challenge for clinical trial design. J Scleroderma Relat Disord 2018;3:170-4.

13. Herrick AL, Roberts C, Tracey A, Silman A, Anderson M, Goodfield M, et al. Lack of agreement between rheumatologists in defining digital ulceration in systemic sclerosis. Arthritis Rheum 2009;60:878-82.

14. Khanna D, Furst DE, Clements PJ, Allanore Y, Baron M, Czirjak L, et al. Standardization of the modified Rodnan skin score for use in clinical trials of systemic sclerosis. J Scleroderma Relat Disord 2017;2:11-8.

J Rheumatol 2019;46:548-9; doi:10.3899/jrheum.180975

Personal non-commercial use only. The Journal of Rheumatology Copyright (C) 2019. All rights reserved. 